\title{
Acceso abierto al conocimiento
}

\author{
Jorge Hernando Bautista-Ruiz $\mathrm{PhD}$ (c) \\ Docente UFPS
}

La dinámica del conocimiento científico en el mundo actual, impone como necesidad dar a conocer los resultados de las investigaciones de manera casi inmediata; lo que pone en desventaja la forma tradicional de publicación (en papel), la cual presenta limitantes como la demora en la comunicación del nuevo conocimiento, y un bajo nivel de circulación y accesibilidad a los manuscritos; esto hace que trabajos valiosos no sean conocidos por la comunidad científica en otras latitudes, reduciendo la visibilidad y el factor de impacto de los mismos; que son medidos por el Índice Nacional de Publicaciones Seriadas, Científicas y Tecnológicas Colombianas - Publindex, para la clasificación de las revistas de carácter científico, y que están directamente asociados con el nivel de accesibilidad de la publicación y el número de citas de los artículos publicados en la revista en un periodo de tiempo determinado respectivamente.

En sinergia con esta dinámica desde hace varios años se ha venido aprovechando el desarrollo de las Tecnologías de la Información y la Comunicación, para aplicarlo a la difusión del conocimiento científico a través de la creación de las primeras publicaciones periódicas electrónicas; este nuevo medio permitió solucionar los inconvenientes de la publicación tradicional en términos de agilidad en los procesos de publicación, circulación y accesibilidad antes mencionados, contribuyendo a mejorar de forma considerable la visibilidad y factor de impacto de las Revistas Científicas; sumado a ello desde el año 2001 se promovió el acceso abierto a la literatura científica denominado "El Open Acces”, el cual permitía acceso a contenidos científicos sin costos económicos asociados.

Esta cultura de acceso abierto permitió el diseño de herramientas para facilitar el manejo de los procesos editoriales de las revistas científicas, impulsando la calidad de la publicación y contribuyendo al bien público a escala global, mediante el aumento del número de lectores, visibilidad e impacto en la comunidad científica; entre dichas herramientas se destaca la aplicación "Open Journal Systems" (OJS), que es una solución de código abierto que permite administrar de forma más eficiente todos los procesos editoriales de una revista científica. Esta plataforma fue lanzada en el año 2002 en la Universidad de British Columbia, como resultado de una iniciativa de investigación y desarrollo de un proyecto de conocimiento público y ha sido empleada por la mayoría de las publicaciones científicas seriadas para administrar sus contenidos; además abarca todos los procesos de edición desde la recepción del manuscrito hasta su publicación. Lo más importante de una revista alojada en el OJS es la posibilidad de acceder a sus contenidos desde cualquier lugar del mundo; lo que permite aumentar la visibilidad tanto de la revista como de los artículos allí publicados, lo que por ende facilita el reconocimiento de los autores.

En consonancia con estas tendencias, que no son nuevas, la Revista RESPUESTAS a partir del primer semestre de 2015 establecerá su proceso de transición editorial a través del OJS y mantendrá su política de acceso abierto a todos los contenidos publicados. En esta nueva etapa la Revista se consolidará en un ámbito científico de mayor amplitud para sí dar a conocer a un mayor número de lectores los trabajos de investigación allí publicados. 